\title{
Caracterização morfológica de Alternaria alternata associado a sementes de alface americana cv. 'Astra'
}

\author{
Paulo Henrique Pereira Costa Muniz ${ }^{1}$, Marina Gabriela Marques ${ }^{1}$, Gustavo Henrique Silva \\ Peixoto ${ }^{1}$, Kamila Gabriela Simão ${ }^{1}$, Daniel Diego Costa Carvalho ${ }^{1}$
}

${ }^{1}$ Universidade Estadual de Goiás, Câmpus Ipameri, Ipameri, Goiás, Brasil. E-mail: paulohenrique.muniz1@gmail.com, magabimarques@hotmail.com, gugspeixoto@gmail.com, kamilagabrielasimao@hotmail.com, daniel.carvalho@ueg.br

Recebido: 28/06/2017; Aceito: 06/12/2017

\begin{abstract}
RESUMO
A mancha de alternaria, cujo agente causal é o fungo, Alternaria spp., encontra-se distribuída pelas áreas de plantio de hortaliças no Brasil onde frequentemente a doença é veiculada por sementes contaminadas. Neste sentido, a qualidade sanitária e fisiológica de sementes é fundamental para o estabelecimento uniforme das culturas em campo, sendo um dos fatores que garantem o sucesso da produção da olerícola. O objetivo deste trabalho foi caracterizar o fungo Alternaria associado a sementes de alface americana cv. 'Astra'. A análise de incidência foi realizada pelo método blotter test, empregando-se o delineamento inteiramente casualizado (DIC), com quatro repetições $\left(50\right.$ sementes gerbox $\left.{ }^{-1}\right)$. Após sete dias a $20^{\circ} \mathrm{C}\left( \pm 2{ }^{\circ} \mathrm{C}\right)$ e foto período de $12 \mathrm{~h}$, a avaliação foi realizada examinando-se individualmente todas as sementes em microscópio estereoscópio para a confecção de lâminas semipermanentes, a qual foi realizada mediante a remoção de micélio e estruturas fúngicas encontradas sobre as sementes infectadas, para caracterização e identificação da espécie do fungo incidente. Os conídios encontrados apresentaram dimensões de 13,7 - 25,7 x 6,2 - 12,4 $\mu \mathrm{m}(18,6$ x 8,8 $\mu \mathrm{m})$, formato de pera invertida, com coloração marrom clara e, cujos bicos apresentaram dimensões de 2,2 - 11,0 $\mu \mathrm{m}(4,7 \mu \mathrm{m})$. Estas características discriminaram o fungo como A. alternata.
\end{abstract}

Palavras-chave: horticultura, micologia, taxonomia de fungos.

\section{Morphological characterization of Alternaria alternata associated on iceberg lettuce seeds cv. 'Astra'}

\begin{abstract}
The Alternaria spot disease, whose causal agent is the fungus Alternaria spp, is distributed throughout the vegetable growing areas in Brazil, where the disease is often transmitted by contaminated seeds. Therefore, the sanitary and physiological quality of seeds is fundamental for the uniform establishment of the crops in the field, being one of the factors that ensure success of the vegetable production. The objective of this work was to characterize the Alternaria fungus occurring in American lettuce seeds cv. 'Astra'. The incidence analysis was performed by the blotter test method, using a completely randomized design (CRD), with four replicates (50 seeds per gerbox). After seven days at $20^{\circ} \mathrm{C}\left( \pm 2{ }^{\circ} \mathrm{C}\right)$ and $12 \mathrm{~h}$ photoperiod, the evaluation was performed by examining all seeds in a stereoscope microscope aiming to the making semi-permanent slides mountings, which was performed by removing mycelium and fungal structures found on the infected seeds, to characterize and identify the incident fungal species. The conidia found exhibit $13.7-25.7$ x $6.2-12.4 \mu \mathrm{m}(18.6$ x $8.8 \mu \mathrm{m})$, inverted pear shape, with light brown coloring and, the conidia beak measurements were $2.2-11.0 \mu \mathrm{m}(4.7 \mu \mathrm{m})$. These characteristics discriminated the fungus as A. alternata.
\end{abstract}

Key words: horticulture, mycology, fungi taxonomy. 
A alface (Lactuca sativa L.) pertencente à família Asteraceae, originária da região do Mediterrâneo, é uma planta herbácea, muito delicada, com um caule diminuto, não ramificado, ao qual se prende às folhas (FILGUEIRA, 2008). Atualmente, é uma das hortaliças folhosas mais cultivadas e consumidas em diversos países, sendo considerada a mais importante na alimentação do brasileiro (NASCIMENTO et al., 2011). Sua expressividade econômica na agricultura nacional é assegurada, principalmente por garantir o fluxo econômico e a sustentabilidade para pequenas e médias propriedades rurais, gerando até cinco empregos diretos por hectare (ALENCAR et al., 2012; LEITE et al., 2016). No Brasil são produzidas em média cerca de 1,5 milhão de toneladas de alface por ano, cultivadas em uma área de 91.454 hectares, movimentando em média $\mathrm{R} \$ 8$ bilhões somente no varejo (ABCSEM, 2016).

A cultura da alface é propagada por meio de sementes, cuja qualidade, principalmente fisiológica e sanitária, é fundamental para garantir o rápido estabelecimento e uniformidade no campo, condicionantes para o sucesso produtivo desta olerícola (NASCIMENTO et al., 2011).

No que se trata à disseminação de patógenos, estudos demonstram que a semente é um veículo de propagação de doenças para a lavoura, principalmente devido à possibilidade de introdução de doenças a longas distâncias (CORRÊA et al., 2008; CARVALHO et al., 2014a). Assim, a ocorrência, de patógenos em sementes de alface como nas demais hortaliças ocasiona efeitos diretos sobre o vigor e rendimento em campo, podendo provocar consideráveis perdas no sistema de produção (NASCIMENTO et al., 2011; MACHADO, 2012; PAIVA et al., 2016).

Dentre os patógenos que podem associar-se às sementes, a incidência do fungo Alternaria spp. em sementes de alface ocasiona necrose do cotilédone e hipocótilo, além de tombamento das mudas (TÖFOLI; DOMINGUES, 2006). As plântulas obtidas nessas condições geralmente morrem ou sofrem enfezamento (MACHADO, 2012). Em plantas adultas os sintomas da alternariose caracterizam-se por manchas necróticas em folhas, pecíolos, flores e inflorescência que acarretam o tombamento da planta (TÖFOLI et al., 2015).

Diversos estudos demonstram que a incidência do fungo em sementes de olerícolas diminuem sua qualidade fisiológica (PEDROSO et al., 2010). Segundo Pereira et al. (2005), a presença de Alternaria alternata, A. radicina e A. dauci, com percentual de incidência em média de $30 \%$, foram determinantes para a diminuição da qualidade fisiológica de sementes de coentro. O fungo Alternaria influi decisivamente na qualidade final das sementes, por expressar microtoxinas que prejudicam o desenvolvimento fisiológico (JAJOR et al., 2012).
A correta identificação do patógeno consiste no primeiro passo para realização do manejo da doença por ele causada, pois auxilia em novas estratégias de controle e nas corretas recomendações de produtos a serem aplicados às sementes (CARVALHO et al., 2014b). Neste contexto, a caracterização morfológica de patógenos auxilia na verificação da diversidade dos fungos e realização de estudos taxonômicos e de identificação do agente causal que atacam determinado hospedeiro (LAZAROTTO et al., 2014). No caso específico do gênero Alternaria, ocorrente em diversos hospedeiros, as características morfológicas mais importantes para a caracterização e a consequente identificação das espécies são as dimensões dos conídios e dos bicos dos conídios que cada uma apresenta (RODRIGUES et al., 2010; ALHUSSAEN, 2012; VASCONCELOS et al., 2014).

No Brasil, há carência de pesquisas envolvendo a correta identificação morfológica de espécies de Alternaria spp., bem como a incidência e associação desse patógeno à sementes de hortaliças, dificultando os estudos sobre as consequências que esta associação pode trazer nos diferentes estádios da cultura (PAIVA et al., 2016). Neste contexto, o objetivo desse trabalho foi caracterizar o fungo Alternaria alternata ocorrente em sementes de alface americana cv. 'Astra', por meio de marcadores morfológicos

A análise sanitária das sementes foi realizada pelo método blotter test (BRASIL/MAPA, 2009). Tal metodologia consistiu em utilizar caixas acrílicas transparentes do tipo gerbox $(11 \times 11 \times 3,5 \mathrm{~cm})$ previamente esterilizadas com álcool 70\%; em seguida, foram colocados dois papéis mata-borrão, previamente umedecidos com água destilada esterilizada a 2,5 vezes seu peso. Em seguida, sementes não tratadas de alface americana cv. 'Astra' foram semeadas nas caixas gerbox. O delineamento utilizado foi o inteiramente casualizado (DIC) com quatro repetições (50 sementes gerbox $^{-1}$ ) (PEDROSO et al., 2013). Após 7 dias a $20{ }^{\circ} \mathrm{C}$ $\left( \pm 2{ }^{\circ} \mathrm{C}\right)$ e fotoperíodo de 12 horas a avaliação da incidência dos patógenos foi realizada examinando-se individualmente todas as sementes em microscópio estereoscópio, estimando-se o percentual de incidência.

Para confirmação do gênero fúngico presente nas sementes e caracterização morfológica, foram confeccionadas lâminas semipermanentes para observações microscópicas, contendo o material biológico removido diretamente do tecido da semente infectada, utilizando ácido lático como meio de montagem para as lâminas (LIMA et al., 2004). Em seguida, realizaram-se o registro de imagens e a caracterização morfológica dos conídios e de suas estruturas, pela obtenção de 30 medidas de cada componente (VASCONCELOS et al., 2014), utilizando o microscópio de luz Leica DM500, com auxílio do 
programa LAS EZ $2.0(100 \mathrm{x})$. As preparações microscópicas foram depositadas no Laminário Fitopatológico da Universidade Estadual de Goiás (UEG).

Os resultados relativos às medidas de comprimento $\mathrm{e}$ largura dos conídios foram submetidos à análise de variância para obtenção do coeficiente de variação destas medidas, com auxílio do programa estatístico SISVAR 5.3 (FERREIRA, 2011). Após mensuração dos conídios de Alternaria, verificou-se que estes possuíam dimensões de 13,7 - 25,7 x 6,2 - 12,4 $\mu \mathrm{m}(18,6$ × 8,8 $\mu \mathrm{m})$ (Tabela 1), sendo que $70 \%$ dos conídios possuíam bicos com dimensões de 2,2 - 11,0 $\mu \mathrm{m}(4,7 \mu \mathrm{m})$. Os mesmos apresentaram-se em formato de pera invertida, ovoides ou elipsoides com coloração marrom clara, com a presença de septos longitudinais e transversais (Figuras 1A e 1B); características que indicam que $A$. alternata seja a espécie ocorrente nas sementes de alface cv. 'Astra'.

$\mathrm{Na}$ análise das dimensões de A. alternata, verificouse que o coeficiente de variação para o comprimento e largura dos conídios foi de $17,77 \%$ e $19,02 \%$, respectivamente. A incidência de A. alternata verificada nas sementes foi de $44,92 \%$. As sementes naturalmente infectadas foram totalmente colonizadas pelo patógeno, sendo cobertas por uma colônia de coloração cinza escuro, aéreo, variável em densidade, com produção de conidióforos diretamente sobre as sementes que se estendia também pelo papel de filtro colocado nas caixas gerbox, impedindo as sementes de germinarem (BRASIL/MAPA, 2009). Adicionalmente, para as comparações, foram utilizadas as características morfológicas de outras espécies de Alternaria ocorrentes no mesmo hospedeiro e de A. alternata ocorrentes em hospedeiros diferentes. Os resultados das dimensões dos conídios de Alternaria deste trabalho são semelhantes aos que Van der Waals et al. (2011), Vasconcelos et al. (2014) e Carvalho et al. (2008) obtiveram para A. alternata, em folhas de batata, tubérculo de batata e frutos de citros, respectivamente. Estes autores encontraram conídios com dimensões de 19,7 - 60,0 x 5,0 - 18,0 $\mu \mathrm{m}$ e comprimento do bico do conídio de 2,5 - 11,9 $\mu \mathrm{m}$ (Tabela 1).

Tabela 1. Características morfológicas de diferentes espécies do gênero Alternaria, em diferentes hospedeiros, incluindo Alternaria alternata, encontrada em sementes de alface americana cv. 'Astra' (Ipameri, GO, 2017).

\begin{tabular}{|c|c|c|c|c|}
\hline \multirow{2}{*}{ Espécie de Alternaria } & \multirow{2}{*}{ Hospedeiro / órgão da planta } & \multicolumn{2}{|c|}{ Tamanho do conídio $(\mu \mathrm{m})$} & \multirow{2}{*}{$\begin{array}{c}\text { Tamanho do bico } \\
(\mu \mathrm{m})\end{array}$} \\
\hline & & Comprimento & Largura & \\
\hline A. grandis $^{(1)}$ & Solanum tuberosum / folha & $102,0-184,0$ & $14,0-17,0$ & $135,0-206,0$ \\
\hline A. solani $i^{(1)}$ & S. tuberosum / folha & $85,0-100,0$ & $18,0-22,0$ & $83,0-110,0$ \\
\hline A. alternata $^{(2)}$ & S. tuberosum / folha & $20,0-60,0$ & $9,0-18,0$ & $*$ \\
\hline A. alternata $^{(3)}$ & S. tuberosum / tubérculo & $19,7-45,4$ & $8,5-18,0$ & $2,6-11,9$ \\
\hline A. alternata $^{(4)}$ & Citrus / fruto & $22,5-35,0$ & $5,0-10,0$ & $2,5-7,5$ \\
\hline A. chicorri $^{(5)}$ & Cichorium endivia / folha & $46,0-79,0$ & $10,0-16,0$ & $*$ \\
\hline A. brassicicola $^{(6)}$ & Brassicae oleraceae / sem. & $18,0-130,0$ & $8,0-20,0$ & $*$ \\
\hline A. brassicae ${ }^{(6)}$ & B. oleraceae / sementes & $75,0-350,0$ & $20,0-30,0$ & $*$ \\
\hline A. $\operatorname{sonchi}^{(7)}$ & Lactuca sativa / folhas & $60,0-91,0$ & $12,0-19,0$ & 22,0 \\
\hline A. alternata ${ }^{(8)}$ & Lactuca sativa / sementes & $13,7-25,7$ & $6,2-12,4$ & $2,2-11,0$ \\
\hline
\end{tabular}

BRASIL/MAPA, (2009); ${ }^{(7)}$ Dingley (1970); ${ }^{(8)}$ Espécie encontrada em sementes de alface americana cv. 'Astra'. *Não relatado pelos autores.
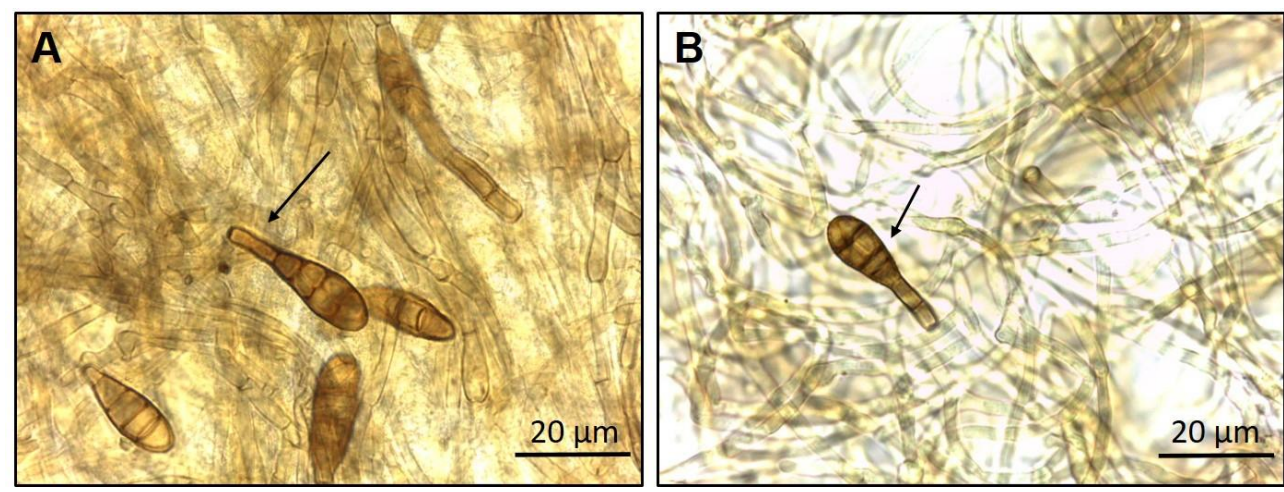

Figura 1. Conídios de Alternaria alternata provenientes de sementes de alface cv. 'Astra' (Ipameri, GO, 2017). A) conídios de coloração marrom clara, em forma de clava ou pera invertida, ovoides ou elipsoides. A seta mostra o bico curto, com comprimento inferior a um terço do corpo do conídio; B) a seta mostra o septo transversal do conídio de A. alternata. 
De forma oposta, os resultados das dimensões dos conídios de A. grandis e A. solani (85,0 - 184,0 x 14,0$22,0 \mu \mathrm{m})$ e dos bicos dos conídios $(83,0-206,0 \mu \mathrm{m})$ verificados em folhas de batata por Rodrigues et al. (2010) são muito diferentes das medições obtidas de conídios de Alternaria em sementes de alface. De forma análoga, embora as espécies A. chicorri, A. brassicae e A. brassicicola sejam relatadas incidindo em sementes de hortaliças, os conídios dessas espécies possuem maior comprimento, isto é, 46,0 - 350,00 x 8,0 - 30,0 $\mu \mathrm{m}$ (LIMA et al., 2003, BRASIL/MAPA, 2009). A espécie A. sonchi, considerado agente etiológico da 'mancha de alternaria' em alface, também pode ser refutado, uma vez que seus conídios medem 60,0 - 91,0 x 12,0 - 19,0 $\mu \mathrm{m}$, além de possuir bicos de tamanho muito maior: 22,0 $\mu \mathrm{m}$ (DINGLEY, 1970).

Durante a análise estatística das dimensões dos conídios de A. alternata, ressaltou-se a variabilidade das medidas obtidas; o coeficiente de variação, para o comprimento e largura dos conídios foi de $17,77 \%$ e 19,02\%, respectivamente. A espécie A. alternata f. sp. citri cuja variabilidade dos dados referentes às medidas de todas as estruturas do fungo não passou de 5,1\% (CARVALHO et al., 2008). Estes valores sugerem que os conídios obtidos diretamente do tecido vegetal do hospedeiro, sem posterior repicagem para meio de cultura, tendem a ser mais variáveis, possivelmente devido às variações ambientais inexistentes no cultivo in vitro (VASCONCELOS et al., 2014).

No Brasil, o registro da mancha de alternaria em alface refere-se à espécie $A$. sochi como o principal agente causal (KRAUZI-SAKATE et al., 2016). No entanto, as características descritas para $A$. sochi não corroboram às verificadas para o fungo encontrado no presente trabalho, pois a A. alternata encontrada nas sementes de alface apresentaram conídios com septos longitudinais e transversais, enquanto que $A$. sonchi apresenta conídios com maiores dimensões e somente septos transversais e raros septos longitudinais, não apresentando apêndices no conídio (ELLIS, 1971; LIMA et al., 2003).

Baseando-se nas características morfológicas dos conídios analisados no presente trabalho, verificou-se tratar-se da ocorrência de A. alternata em sementes de alface americana cv. 'Astra'.

\section{Referências Bibliográficas}

ABCSEM. ASSOCIAÇÃO BRASILEIRA DO COMÉRCIO DE SEMENTES E MUDAS. Projeto para levantamento dos dados socioeconômicos da cadeia produtiva de hortaliças no Brasil. Campinas-SP: ABCSEM, 2016. 16 p. Disponível em:

http://www.abcsem.com.br/upload/arquivos/O_mercado_de_f olhosas_Numeros_e_Tendencias_-_Steven.pdf $>$ Acesso em: 12 jun. $\overline{2017 .}$
ALENCAR, T. A.; TAVARES, A. T.; CHAVES, P. P. N.; FERREIRA, T. A.; NASCIMENTO, I. R. Efeito de intervalos de aplicação de urina bovina na produção de alface em cultivo protegido. Revista Verde. Mossoró-RN, v. 7, n. 3, p. 53-67, 2012.

ALHUSSAEN, K. M. Morphological and physiological characterization of Alternaria solani isolated from tomato in Jordan Valley. Research Journal of Biological Sciences, Karaman, v. 7, n. 8, p. 316-319, 2012.

BRASIL/MAPA. MINISTÉRIO DA AGRICULTURA, PECUÁRIA E ABASTECIMENTO. Regras para análise de sementes. Brasília-DF: Secretaria de Defesa Agropecuária. Mapa/ACS, 2009, 399 p.

CARVAlHO, D. D. C.; ALVES, E.; BATISTA, T. R. S.; CAMARGOS, R. B.; LOPES, E. A. G. L. Comparison of methodologies for conidia production by Alternaria alternata from citrus. Brazilian Journal of Microbiology, São PauloSP, v. 39, n. 4, p. 792-798, 2008.

CARVALHO, D. D. C.; JUNIOR, M. L.; MARTINS, I.; INGLIS, P. W.; MELLO, S. C. M. Biological control of Fusarium oxysporum f. sp. phaseoli by Trichoderma harzianum and its use for common bean seed treatment. Tropical Plant Pathology, Brasília-DF, v. 39, p. 384-391, 2014a.

CARVALHO, D. D. C.; OLIVEIRA, A. M. E.; LAGO, H. M. S.; RODRIGUES, F. Incidência de Bipolaris bicolor em sementes de sorgo granífero no Brasil. Revista Brasileira de Milho e Sorgo, Sete Lagoas-MG, v. 13, n.2, p. 240-247, 2014b.

CORRÊA, B. O.; MOURA, A. B.; DENARDIN, N. D.; SOARES, V. N.; SCHÄFER, J. T.; LUDWIG, J. Influence of bean seed microbiolization on the transmission of Colletotrichum Lindemuthianum (Saac and Magn.). Revista Brasileira de Sementes, Londrina-PR, v. 30, n. 2, p. 156-163, 2008.

DINGLEY, J. M. Records of fungi parasict on plants in New Zealand 1966-68. New Zeland Journal of Agricultural Reserch, Wellington, v. 13, n. 1, p. 325-337, 1970.

ELLIS, M. B. Dematiaceous Hyphomycetes. Kew: Commonwealth Mycological Institute, 1971. 608 p.

FERREIRA, D. F. Sisvar: a computer statistical analysis system. Ciência e Agrotecnologia, Lavras-MG, v. 35, n. 6, p. 1039-1042, 2011.

FILGUEIRA, F. A. R. Novo Manual de Olericultura: agrotecnologia moderna na produção e comercialização de hortaliças, 3 ed. Viçosa-MG: Universidade Federal de Viçosa, 2008. $421 \mathrm{p}$.

JAJOR, E.; KOZLOWSKA, M.; WOJTOWICZ, M. Prevalence of fungi of the genus Alternaria on rape siliques and seeds depending on weather conitions. Progress in Plant Protection, Póznan, v. 52, n. 4, p. 1011-1015, 2012.

KRAUSE-SAKATE, R.; PAVAN, M. A.; MOURA, M. F.; KUROZAWA, C. Doenças da alface. In: KIMATI, H.; AMORIM, L.; REZENDE, J. A. M.; BERGAMIN FILHO, A.; CAMARGO, L. E. A. Manual de Fitopatologia: Doenças 
das plantas cultivadas. 5. ed. Ouro Fino: Agronômica Ceres, v. 2, 2016. p. 33-40.

LAZAROTTO, M.; MILANESI, P. M.; MUNIZ, F. B.; REINIGER, L. R. S.; BELTRAME, R.; HARAKAVA, R.; BLUME, E. Morphological and molecular characterization of Fusarium spp. pathogenic to pecan tree in Brasil. Genetics and Molecular Research, Ribeirão Preto-SP, v. 13, n. 4, p. 9390-9402, 2014.

LEITE, D.; MIGLIAVACCA, R. A.; MOREIRA, L. A.; ALBRECHT, A. J. P.; FAUSTO, D. A. Viabilidade econômica da implantação do sistema hidropônico para alface com recursos do PRONAF em Matão-SP. Revista iPecege, Piracicaba-SP, v. 2, n. 1, p. 57-65, 2016.

LIMA, C. S.; SOUZA, P. E.; BOTELHO, A. O. Rust fungi of the Pucciniaceae family on medicinal plants. Fitopatologia Brasileira, Brasília-DF, v. 29, n. 5, p. 499-503, 2004.

LIMA, M. L. P.; REIS, A.; LOPES, C. A. Patogenicidade de Alternaria cichorii sobre Espécies da Família Asteraceae no Brasil. Fitopatologia Brasileira, Brasília-DF, v. 28, n. 6, p. 682-685, 2003.

MACHADO, J. C. Patologia de sementes: significado e atribuições. In: CARVALHO, N. M.; NAKAGAWA, J. (Eds.). Sementes: ciência, tecnologia e produção. JaboticabalSP: Funep, 2012. p.524-590.

MARINO, R. H. Incidência de fungos em sementes de Phaseolus vulgaris L. provenientes do estado de Sergipe. Revista Brasileira de Ciências Agrárias, Recife-PE, v. 3, n. 1, p. 26-30, 2008.

NASCIMENTO, W. M.; DIAS, D. C. F. S.; SILVA, P. P. Qualidade da semente e estabelecimento de plantas de hortaliças no campo. In: NASCIMENTO, W. M. (Ed.). Hortaliças: tecnologia de produção de sementes. Brasília-DF: Embrapa Hortaliças, 2011. p. 79-106.

PAIVA, C. T. C.; SILVA, J. P.; DAPONT, E. C.; ALVES, C. Z.; CARVALHO, M. A. C. Qualidade fisiológica e sanitária de sementes comerciais de alface e repolho. Revista de Ciências Agroambientais, Alta Floresta-MT, v. 14, n. 1, p. 53-59, 2016.
PEDROSO, D. C.; MENEZES V. O.; MUNIZ, M. F. B.; PIVETA, G.; TUNES, L. M.; MULLER, J.; MENEZES, N. L. Métodos de inoculação de Alternaria alternata e A. dauci em sementes de salsa e sua influência na qualidade fisiológica. Revista Brasileira de Sementes, Londrina-PR, v. 32, n. 3, p. 79-85, 2010.

PEDROSO, D. C.; MUNIZ, M. F. B.; TUNES, L. M.; MULLER, J.; JUNGES, E.; SANTOS, R. F. Influência de Alternaria alternata e A. dauci na qualidade de sementes de coentro. Revista Brasileira de Ciências Agrárias, Recife-PE, v. 8 , n. 4 , p. $563-569,2013$.

PEREIRA, R.S.; MUNIZ, M.F.B.; NASCIMENTO, W.M. Aspectos relacionados à qualidade de sementes de coentro. Horticultura Brasileira, Brasília-DF, v. 23, n. 3, p.703-706, 2005.

RODRIGUES, T. T. M. S.; BERBEE, M. L.; SIMMONS, E. G.; CARDOSO, C. R.; REIS, A.; MAFFIA, L. A.; MIZUBUTI E. S. G. First report of Alternaria tomatophila and $A$. grandis causing early blight on tomato and potato in Brazil. New Disease Reports, Wellesbourne, v. 22, n. 1, p. 28, 2010.

TÖFOLI, J. G.; DOMINGUES, R. J. Alternarioses em hortaliças: Sintomas, etiologia e manejo integrado. Biológico, São Paulo-SP, v. 66, n. 1, p. 23-30, 2006.

TÖFOLI, J. G.; DOMINGUES, R. J.; FERRARI, J. T. Alternaria spp. In Oleraceous: Symptons, Etiology, Management and Fungicides. Biológico, São Paulo-SP, v. 77, n. 1, p. 21-34, 2015.

VASCONCELOS, C. V.; SILVA, D. C.; CARVALHO, D. D. C. Ocorrência de Alternaria alternata (Fr.:Fr.) Keissl. em tubérculos de batata, no Brasil. Pesquisa Agropecuária Tropical, Goiânia-GO, v. 44, n. 2, p. 219-222, 2014.

VAN DER WAALS, J. E.; PITSI, B. E.; MARAIS, C.; WAIRURI, C. K. First report of Alternaria alternata causing leaf blight of potatoes in South Africa. Plant Disease, Saint Paul, v. 95, n. 3, p. 363, 2011. 